\title{
Qualitätskriterien - effektiv und einfach umsetzbar
}

\author{
Jürg Schlup \\ Dr. med., Präsident der FMH
}

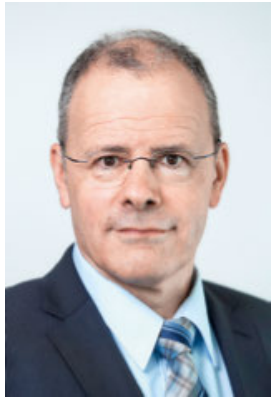

In gut zwei Jahren läuft die befristete Zulassungseinschränkung für praxisambulant tätige Ärzte und Ärztinnen ab, so dass zurzeit die Diskussion um neue Steuerungsoptionen beginnt. In wenigen Wochen will der Bundesrat einen Bericht mit Antworten zum Postulat 16.3000 "Alternativen zur heutigen Steuerung der Zulassung von Ärztinnen und Ärzten» vorlegen. Im nächsten Sommer dürfte er dann einen Gesetzesentwurf zur Steuerung von Ärztinnen und Ärzten im praxisambulanten Versorgungssektor in Vernehmlassung geben.

Die drei im Parlament diskutierten Steuerungsvarianten dürften jedoch mehr Nebenwirkungen als Wirkungen entwickeln. So wird die kantonale Steuerung der Anzahl praxisambulanter Ärzte innerhalb national vorgegebener Ober- und Untergrenzen vorgeschlagen. Dies würde die kostentreibenden Interessenkonflikte der spitalambulante Leistungen erbringenden Kantone noch verstärken. Zudem orientieren sich Patienten kaum an Kantonsgrenzen, sondern an Versorgungsregionen und urbanen Zentren. Schliesslich ist die Ärztezahl als Messgrösse angesichts neuer Arbeitsmodelle, Teilzeittätigkeit und Elternzeiten heute ungeeignet. Zeitgemäss wären Vollzeitäquivalente. Dazu - wie auch zu vielen anderen Faktoren - fehlen jedoch die unverzichtbaren statistischen Grundlagen.

Auch die zweite Variante mit der Idee regionsspezifisch differenzierter Vergütungen - quasi einem tarifarischen Voralpenzuschlag - geht an der Praxis der Gesundheitsversorgung vorbei. Beispiele von Kantonsgrenzen, an denen sich mehr Praxen auf der Seite mit dem niedrigeren Taxpunktwert ansiedeln, zeigen, dass wirksame Preisunterschiede mindestens $20 \%$ betragen müssten und folglich teuer wären. Zudem müssten diese Preise langfristig stabil bleiben, um den Investitionszyklen für Arztpraxen zu entsprechen, sonst würde sich die Versorgung im ländlichen Raum trotz Zuschlägen nicht verbessern, die Kosten aber würden sich erhöhen.

Die dritte Variante - eine Lockerung des Vertragszwangs - zeugt mehr vom Einfluss der Krankenkassenverbände als vom Ziel einer hochwertigen praxis-

ambulanten Versorgung: Die freie Arztwahl würde obligatorisch eingeschränkt; die Kostenkriterien der Kassen würden die Arzt-Patient-Beziehung belasten und Umgehungsbewegungen auf nicht vertragsbetroffene Spitalsektoren auslösen.

Unser Vorschlag fokussiert darum auf vier wirksame, einfach umsetzbare, kumulativ und landesweit zu erfüllende Qualitätskriterien. Mit diesen Kriterien kann nicht nur eine Reduktion der Zulassungen in qualitativ wünschenswerter Weise, sondern auch eine bedarfsgerechte Verteilung der ärztlichen Fachdisziplinen erreicht werden:

- Nachweis einer mindestens dreijährigen fachspezifischen ärztlichen Tätigkeit in der für die Zulassung beantragten Fachdisziplin an einer anerkannten

\section{Die FMH schlägt vier wirksame, einfach} umsetzbare, kumulativ und landesweit zu erfüllende Qualitätskriterien für die Zulassung vor.

Schweizer Weiterbildungsstätte. Damit würden überproportionale Zulassungen einzelner Spezialdisziplinen durch die begrenzten Stellen mit fachspezifischer Tätigkeit verhindert.

- Nachweis der für die Tätigkeitsregion erforderlichen Sprachkompetenz durch eine in der Schweiz absolvierte Sprachprüfung vor Antritt der ärztlichen Tätigkeit: An dieser Hürde scheitern in Deutschland bis zu 40\% der ausländischen Bewerber.

- Nachweis eines dem mitteleuropäischen Standard äquivalenten Medizinstudiums von mind. 5500 Stunden theoretischem und praktischem Unterricht.

- Nachweis der aktuellen jährlichen Fortbildung. Dies ist mittels Fortbildungsdiplom für alle 46 Fachgebiete einfach überprüfbar.

Bei einer gleichzeitig gezielten Förderung von Praxisassistenzstellen in Regionen mit geringer Hausarztdichte wäre eine praxistaugliche Lösung ohne zusätzlichen Verwaltungsaufwand erreicht. Die FMH gibt gerne Auskünfte zu ihren Erfahrungen mit solchen Förderungen und den Kriterien im Detail - und bietet Hand für eine Qualitätslösung! 